\title{
Real-Time RT-PCR for the Detection of Lyssavirus Species
}

\author{
A. Deubelbeiss, M.-L. Zahno, M. Zanoni, D. Bruegger, and R. Zanoni \\ Institute of Virology and Immunology, 3012 Berne, Switzerland \\ Correspondence should be addressed to R. Zanoni; zanoni@vetsuisse.unibe.ch \\ Received 21 July 2014; Revised 19 September 2014; Accepted 24 September 2014; Published 16 October 2014 \\ Academic Editor: Masanori Tohno
}

Copyright (C) 2014 A. Deubelbeiss et al. This is an open access article distributed under the Creative Commons Attribution License, which permits unrestricted use, distribution, and reproduction in any medium, provided the original work is properly cited.

\begin{abstract}
The causative agents of rabies are single-stranded, negative-sense RNA viruses in the genus Lyssavirus of Rhabdoviridae, consisting of twelve classified and three as yet unclassified species including classical rabies virus (RABV). Highly neurotropic RABV causes rapidly progressive encephalomyelitis with nearly invariable fatal outcome. Rapid and reliable diagnosis of rabies is highly relevant for public and veterinary health. Due to growing variety of the genus Lyssavirus observed, the development of suitable molecular assays for diagnosis and differentiation is challenging. This work focused on the establishment of a suitable real-time RT-PCR technique for rabies diagnosis as a complement to fluorescent antibody test and rabies tissue culture infection test as gold standard for diagnosis and confirmation. The real-time RT-PCR was adapted with the goal to detect the whole spectrum of lyssavirus species, for nine of which synthesized DNA fragments were used. For the detection of species, seven probes were developed. Serial dilutions of the rabies virus strain CVS-11 showed a 100-fold higher sensitivity of real-time PCR compared to heminested RT-PCR. Using a panel of thirty-one lyssaviruses representing four species, the suitability of the protocol could be shown. Phylogenetic analysis of the sequences obtained by heminested PCR allowed correct classification of all viruses used.
\end{abstract}

\section{Introduction}

Rabies diagnosis is based on fluorescent antibody testing (FAT) in brain smears and inoculation of brain suspension either in mouse neuroblastoma cell cultures or intracerebrally in mice as confirmatory assays with high sensitivity and specificity for postmortem diagnosis [1-3]. These techniques are well suited for rapid and reliable routine diagnosis, if brain material is available. For other diagnostic specimens sampled intra vitam in case of clinical suspicion, for example, saliva, cerebrospinal fluid, or skin biopsies, more suited molecular techniques with excellent sensitivity have been developed and validated, mostly targeting the conserved nucleoprotein gene of the lyssavirus genome, for example, [4-10]. Among these, there are protocols both for classical RT-PCR and for realtime RT-PCR, which adds speed, efficiency, contamination safety, and reliability to the technique combined with the potential to quantify the viral load [11,12]. A major advantage offered by these molecular techniques is the characterisation of viral isolates by sequencing the given amplification products followed by phylogenetic or phylogeographic analysis [13-15]. The greatest challenge for these powerful novel techniques is the ever growing spectrum of known lyssavirus species/genotypes (GT), probably all of which having the potential to cause animal and human rabies fatalities $[16,17]$. So far, 12 lyssavirus species and three as yet unclassified species have been identified [17]. The development of classical simple or (hemi)nested RT-PCR methods with particular emphasis on the diagnosis of a broad spectrum of lyssaviruses species has been published in a number of studies [1823]. Also several real-time RT-PCR protocols suited for the broad detection or differentiation of several genotypes have been developed [9, 24-28]. The work of Wakeley et al. [25] is of particular interest for the rabies epidemiology in Europe concerning the European bat lyssaviruses types 1 and 2 [29-32] apart from classical rabies [33, 34]. This assay uses genotype (GT) specific probes and fluorophore signals for direct differentiation of GT1, GT5, and GT6 in a single-tube reaction. The protocol proposed by Nadin-Davis et al. [8] is highly suited for the detection of a broad range of classical rabies viruses.

The goal of this work was, based on a comprehensive review of the rich literature on the theme and adapting thereof, to develop, establish, and validate classical and 
real-time RT-PCR protocols for (intravitam) diagnosis of rabies and molecular-epidemiological characterisation of viral strains with the main emphasis on the growing number of known species/genotypes.

\section{Materials and Methods}

\subsection{Samples}

2.1.1. Cell Culture Supernatant of Viral Strains. All operations with potentially infectious material apart from centrifugation in closed tubes were performed in a class II biological safety cabinet. Frozen cell culture supernatants of rabies virus strains propagated previously on BHK-21 cells (baby hamster kidney cells, ATCC, Manassas, USA) were thawed, centrifuged at $2,000 \mathrm{~g}$ for $10 \mathrm{~min}$ at $4^{\circ} \mathrm{C}$, and filtrated using the Millex-HA $450 \mathrm{~nm}$ Filter (Millipore, Cork, Ireland).

2.1.2. Brain Suspension. Approximately $1 \mathrm{~g}$ of cerebellum, medulla oblongata, and hippocampus from unfixed, freshly obtained brain material was homogenized to a $20 \%$ brain suspension using a mortar and pestle, after addition of approximately $1 \mathrm{~g}$ of quartz sand (Merck KGaA, Darmstadt, Germany) and $5 \mathrm{~mL}$ of Modified Eagle Medium with Earle's salts with $2.2 \mathrm{~g} / \mathrm{L} \mathrm{NaHCO}_{3}$ (Bioswisstec AG, Schaffhausen, Switzerland) supplemented with $5 \%$ penicillin $10,000 \mathrm{IU} / \mathrm{mL}$ (Bioswisstec AG, Schaffhausen, Switzerland) and 20\% foetal calf serum (FCS; PAA Laboratories GmbH, Pasching, Austria). The suspension was then decanted into a $5 \mathrm{~mL}$ tube (5 mL Polystyrene Round-Bottom Tube; BD Biosciences, Erembodegem, Belgium) to let it sediment for 1 hour at $4^{\circ} \mathrm{C}$ and subsequently centrifuged at $1,400 \mathrm{~g}$ for $10 \mathrm{~min}$ and filtrated as above. Frozen brain suspensions from former mouse inoculation tests [39] were handled like frozen cell culture supernatant.

2.1.3. Saliva and Oral Swabs. $500 \mu \mathrm{L}$ of RNA Storage Solution (Ambion, Foster City, USA) was added to $100-200 \mu \mathrm{L}$ of fresh or previously frozen saliva samples or swabs, which were subsequently vortexed (Vortex Genie 2, TEWIS Laborbedarf AG, Berne, Switzerland) for $1 \mathrm{~min}$ and centrifuged in a Biofuge Pico (Heraeus Holding GmbH, Hanau, Germany) at $5,000 \mathrm{rpm}$ for $10 \mathrm{~min}$.

2.1.4. Cerebrospinal Fluid. Cerebrospinal fluid (CSF) samples were diluted 1:2 to $1: 4$ with RNA Storage Solution (Ambion, Foster City, USA).

2.1.5. Skin Biopsies. Skin biopsies were shaved, cut into small pieces with a sterile pair of scissors, and further processed as described for brain suspension.

2.2. Viral Strains and Propagation in Cell Culture. A panel of 31 lyssaviruses (including 4 species) was used to test the diagnostic performance of our PCR (Table 1). Stocks of viral strains were produced in neuroblastoma cells (MNA $42 / 13$ ) as described for the rabies tissue culture infection test (RTCIT, described below). The maintenance medium used for the first passage was Dulbecco's MEM supplemented with $0.5 \%$ neomycin $50 \mathrm{IU} / \mathrm{mL}$ (Bioswisstec AG, Schaffhausen, Switzerland), 5\% tryptose phosphate (BioConcept Ltd. Amimed, Allschwil, Switzerland), 1\% nonessential amino acids (Bioswisstec AG, Schaffhausen, Switzerland), 3\% foetal calf serum, and 1\% L-Glutamine $200 \mathrm{mM}$ (Bioswisstec AG, Schaffhausen, Switzerland) to which $1 \%$ Diethylaminoethyl-Dextran (DEAE-Dextran; Sigma-Aldrich Corporation, St. Louis, USA) was added. Dulbecco's MEM with $10 \%$ foetal calf serum was used for the 3 consecutive passages. Staining of the cells was performed after each passage using Rabies DFA Reagent (Millipore, Livingston, $\mathrm{UK}$ ) as a conjugate.

2.3. FAT and RTCIT. The standard fluorescent antibody test (FAT) was performed with brain tissue samples as previously described [40]. Rabies tissue culture infection test (RTCIT) using four consecutive passages on murine neuroblastoma cells [41-43] was applied to clinical specimens like brain specimens, liquor, saliva, or skin biopsies.

2.4. Primers, Probes, and Synthetic DNA. Primers, probes, and synthetic DNA were obtained from Microsynth (Microsynth $\mathrm{GmbH}$, Balgach, Switzerland). The location of suitable N-directed primers and probes for heminested RTPCR and real-time RT-PCR, respectively, was chosen and evaluated based on published work [8, 18, 22, 25]. Using multiple sequence alignment (ClustalX 2.0.3 program [4446]) of the $\mathrm{N}$ gene region of the available lyssavirus species RABV (33 sequences), LBV (4 sequences), MOKV (5 sequences), DUVV (4 sequences), EBLV-1 (17 sequences), EBLV-2 (14 sequences), ABLV, Aravan, Khujand, Irkut, and West Caucasian bat (each one) from GenBank (National Center for Biotechnology Information and National Library of Medicine, Rockville Pike, USA), variable positions of primers and probes were adjusted with wobble positions for a potentially broader match. Alternatively, primers with single wobbles or substitutions were mixed. Several probes for real-time RT-PCR for broadening the spectrum of detectable species were designed in this work (Table 2). As internal control for conventional RT-PCR, amplification of GAPDH (glyceraldehyde 3-phosphate dehydrogenase) was used [37]. As internal inhibition control for real-time RT-PCR, primers and probes for the amplification of Sendai virus (ATCC, Manassas, USA [38]), which was added to the samples before RNA isolation, were used (Table 2).

Synthetic DNA with a length of 125 bases encompassing positions 48-172 according to the Pasteur virus genome (X03673) of the following lyssavirus species were obtained from Microsynth: Aravan virus (ARAV) EF614259, Khujand virus (KHUV) EF614261, Bokeloh bat lyssavirus (BBLV) JF311903, Australian bat lyssavirus (ABLV) AF418014, Irkut virus (IRKV) EF614260, Lagos bat virus (LBV) EU293110, Mokola virus (MOKV) 293117, Shimoni bat virus (SHIV) GU170201, and West Caucasian bat virus (WCBV) EF614258.

2.5. RNA Extraction. RNA was isolated from $140 \mu \mathrm{L}$ of sample supernatant using the QIAamp Viral RNA Mini Kit 


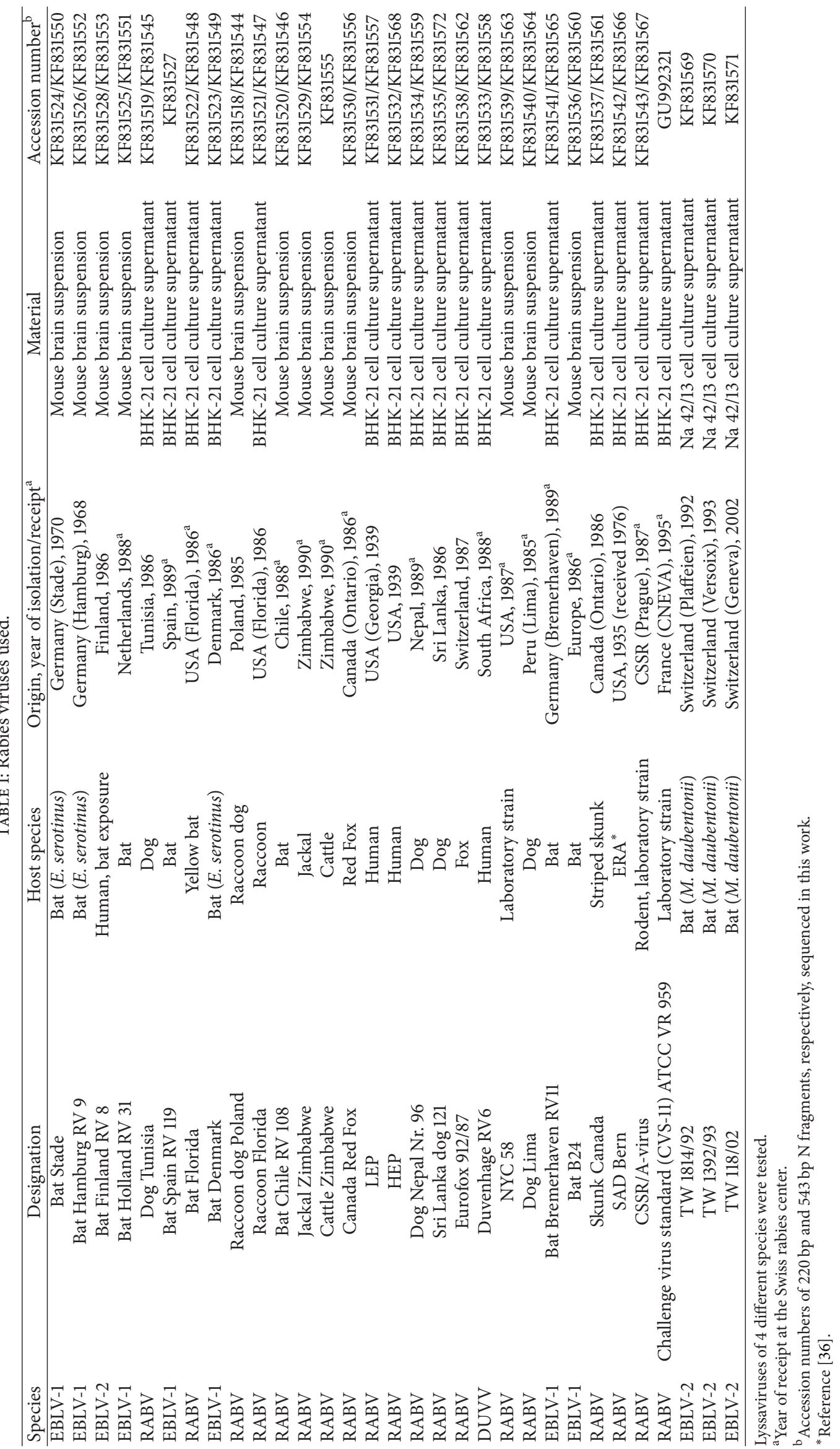




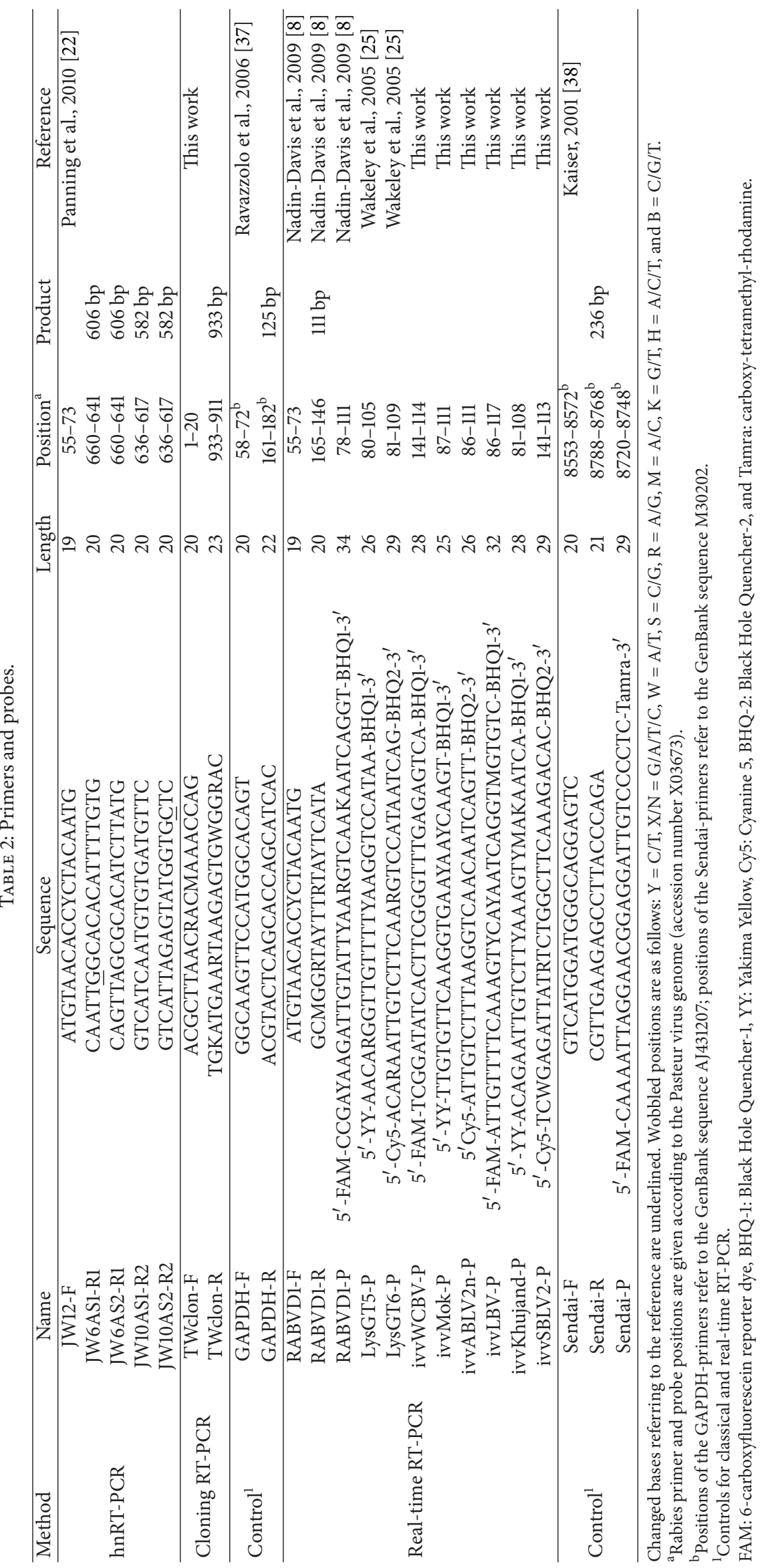


(QIAGEN, Germantown, USA) according to the manufacturer's instructions, except the usage of RNA Storage Solution (Ambion, Foster City, USA) for elution. Extracted RNA was stored at $-20^{\circ} \mathrm{C}$ until use.

2.6. Heminested RT-PCR. Heminested RT-PCR was performed as previously described [22] using the OneStep RTPCR Kit. Briefly, for the first round $3 \mu \mathrm{L}$ of extracted RNA was amplified in the RT-PCR mix prepared according to manufacturer's instructions, supplemented with $0.6 \mu \mathrm{M}$ of each primer (JW12-F, JW6AS1-R1, and JW6AS2-R1; Table 2) and $5 \mathrm{U}$ RNase inhibitor (RNasin Plus, $40 \mathrm{U} / \mu \mathrm{L}$; Promega, Madison, USA), with the following cycling conditions: $50^{\circ} \mathrm{C}$ for $30 \mathrm{~min}$ and $95^{\circ}$ for $15 \mathrm{~min}$ for reverse transcription and subsequent activation of the polymerase, followed by 10 cycles of $95^{\circ} \mathrm{C}$ for $20 \mathrm{~s}, 60^{\circ} \mathrm{C}$ for $30 \mathrm{~s}\left(1^{\circ} \mathrm{C}\right.$ decrease/cycle), followed by $72^{\circ} \mathrm{C}$ for $30 \mathrm{~s}$ and 35 cycles of $95^{\circ} \mathrm{C}$ for $20 \mathrm{~s}, 52^{\circ} \mathrm{C}$ for $30 \mathrm{~s}$, followed by $72^{\circ} \mathrm{C}$ for $30 \mathrm{~s}$. For the second round, the Taq DNA Polymerase Kit (QIAGEN, Germantown, USA) was used with $2 \mu \mathrm{L}$ of the first round product, $2.5 \mu \mathrm{L} 10 \mathrm{x}$ PCR buffer, $1.2 \mathrm{mM}$ final dNTP concentration (Invitrogen, Foster City, USA), $0.4 \mu \mathrm{M}$ of heminested primers (JW12-F, JW10AS1-R2, and JW10AS2-R2; Table 2), $0.5 \mathrm{U}$ of Taq DNA polymerase, and $19.35 \mu \mathrm{L}$ Nuclease Free Water (Ambion, Foster City, USA). Cycling conditions were as follows: $95^{\circ} \mathrm{C}$ for $5 \mathrm{~min}$ followed by 35 cycles of $94^{\circ} \mathrm{C}$ for $20 \mathrm{~s}, 52^{\circ} \mathrm{C}$ for $20 \mathrm{~s}$, followed by $72^{\circ} \mathrm{C}$ for $30 \mathrm{~s}$. Amplifications were performed in a 2720 Thermal Cycler. Internal controls using GAPDH primers, as described in the one-round PCR, were run in parallel in the first round.

2.7. Gel Electrophoresis for Sequencing. Gel electrophoresis was performed using a 1.5\% TAE agarose gel (agarose LE, analytical grade; Promega, Madison, USA) stained with ethidium bromide (Eurobio, Courtaboeuf, France). For sequencing, the desired band was excised under UV light [47].

2.8. Real-Time RT-PCR (NDWD). Real-time RT-PCR (NDWD) adapted from Nadin-Davis et al. and Wakeley et al. $[8,25]$ was performed using the QuantiTect Probe RT-PCR Kit (QIAGEN, Germantown, USA). The $25 \mu \mathrm{L}$ reaction volume consisted of $9.5 \mu \mathrm{L}$ RNase-free water, or $9 \mu \mathrm{L}$ in the multiplex mix, $12.5 \mu \mathrm{L} 2 \mathrm{x}$ QuantiTect RT-PCR Master Mix, $0.25 \mu \mathrm{L}$ of the RABVD1 forward and reverse primer $(0.4 \mu \mathrm{M}$ final concentration each), $0.25 \mu \mathrm{L}$ of the probes RABVD1, LysGT5, and LysGT6 together in the multiplex mix or $0.25 \mu \mathrm{L}$ of the probes ivvWCBV-P, ivvMok-P, ivvABLV2n-P, ivvLBV-P, ivvKhujand-P, and ivvSBLV2-P (Table 2) alone in a mix (all $0.2 \mu \mathrm{M}$ final concentration), $0.25 \mu \mathrm{L}$ of QuantiTect RT Mix, and $2 \mu \mathrm{L}$ of sample RNA. Additionally, for the internal Sendai virus control, $5 \mu \mathrm{L}$ of sample RNA was added to a mix containing $6.625 \mu \mathrm{L}$ of RNase-free water, $12.5 \mu \mathrm{L} 2 \mathrm{x}$ QuantiTect RT-PCR Master Mix, $0.625 \mu \mathrm{L}$ of Sendai forward primer, reverse primer, and probe mix (Sendai-F, Sendai-R, and Sendai-P, $8 \mu \mathrm{M}$; Table 2), and $0.25 \mu \mathrm{L}$ of QuantiTect RT Mix. The reactions were carried out in MicroAmp Fast Optical 96-Well Reaction Plates with Barcode $0.1 \mathrm{~mL}$ (Applied Biosystems, Foster City, USA) in a
7500 Fast Real-Time PCR System v1.3.1 (Applied Biosystems, Foster City, USA) using the following cycling conditions: 1 cycle of $50^{\circ} \mathrm{C}$ for $30 \mathrm{~min}$ and $95^{\circ} \mathrm{C}$ for $15 \mathrm{~min}$ followed by 45 cycles of $95^{\circ} \mathrm{C}$ for $15 \mathrm{sec}$ and $50^{\circ} \mathrm{C}$ for $1 \mathrm{~min}$. Amplification curve analysis was performed using the 7500 Software v2.0.6 (Applied Biosystems, Foster City, USA).

2.9. Cloning for Analytical Sensitivity Analysis. For cloning, a segment of viral genome covering a part of the $\mathrm{N}$ gene (positions 1-933 according to the Pasteur virus genome accession number X03673) of CVS-11 was generated using primers designed for this purpose (TWclon-F and TWclonR, Table 2). Amplification was performed using the OneStep RT-PCR Kit with the master mix consisting of $29.25 \mu \mathrm{L}$ of RNase-free water, $10 \mu \mathrm{L} 5 \mathrm{x}$ QIAGEN OneStep RT-PCR buffer, $0.4 \mathrm{mM}$ of each dNTP, $0.6 \mu \mathrm{M}$ of the cloning primers, $10 \mathrm{U}$ RNasin $(40 \mathrm{U} / \mu \mathrm{L})$, and $2 \mu \mathrm{L}$ of QIAGEN OneStep RT-PCR Enzyme mixed with $5 \mu \mathrm{L}$ of extracted RNA to a total volume of $50 \mu \mathrm{L}$. Amplifications were performed in a Veriti 96-Well Thermal Cycler (Applied Biosystems, Foster City, USA) using the following cycling conditions: $30 \mathrm{~min}$ at $50^{\circ} \mathrm{C}$, followed by $15 \mathrm{~min}$ at $95^{\circ} \mathrm{C}$ and 40 repetitive cycles of $1 \mathrm{~min}$ at $94^{\circ} \mathrm{C}, 1 \mathrm{~min}$ with a temperature gradient from $56^{\circ} \mathrm{C}$ to $46^{\circ} \mathrm{C}$ in steps of $2^{\circ} \mathrm{C}$, and $1 \mathrm{~min}$ at $72^{\circ} \mathrm{C}$. Elongation at $72^{\circ} \mathrm{C}$ was extended for 10 additional minutes in the last cycle. After gel electrophoresis excised PCR fragments with a length of $933 \mathrm{bp}$ were eluted with the QIAquick Gel Extraction Kit (QIAGEN, Germantown, USA) and cloned into the pCR-II-TOPO vector using the TOPO TA Cloning Kit (Invitrogen, Foster City, USA) according to the manufacturer's instructions.

2.10. Sequencing. Sequencing reactions were performed by Microsynth AG, Balgach, Switzerland. For this purpose, reaction mixtures containing $22.5 \mathrm{ng}$ DNA per $100 \mathrm{bp}, 30 \mathrm{pmol}$ of each primer used for the amplification (Table 2), and DEPC treated water (Ambion, Foster City, USA) to a final volume of $15 \mu \mathrm{L}$ were prepared. Sequences were edited using the SeqMan II v5.01 Software (DNASTAR, Madison, USA) and subsequently evaluated using the Clone Manager 9 software (Scientific \& Educational Software, Cary, USA).

2.11. Phylogenetic Reconstruction. The $543 \mathrm{bp}$ nucleotide sequences of the nucleoprotein gene obtained from the heminested PCR reaction, together with additional sequences retrieved from GenBank, were saved in Fasta file format. The sequences were then aligned using the ClustalX 2.0.3 Software and the GeneDoc Software v2.5.0 [48]. Phylogenetic trees were constructed with the MEGA5 software using Kimura 2-parameter distances [35].

\section{Results}

3.1. Cell Culture. Thirty-one lyssaviruses (19x RABV, $7 \mathrm{x}$ EBLV-1, 4x EBLV-2, and 1x DUVV) were analysed in RTCIT. All isolates were viable in murine neuroblastoma cells and could be visualized using Rabies DFA Reagent as a conjugate. 


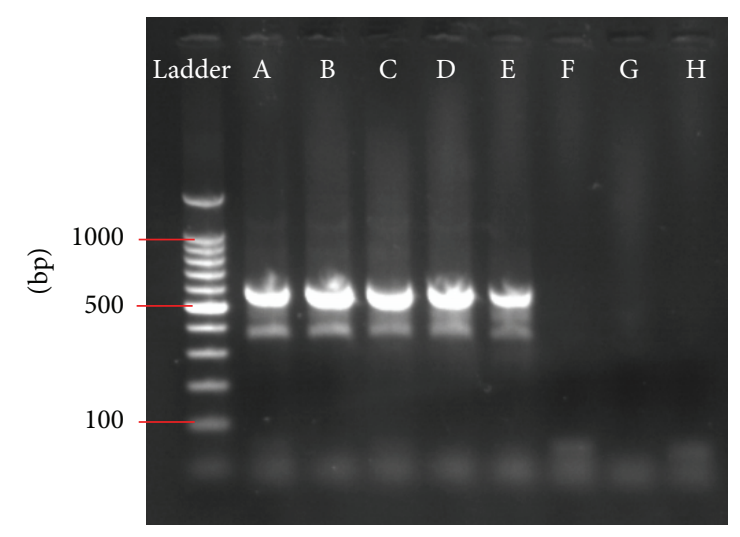

FIGURE 1: Amplification of a CVS-11 serial dilution using hnRTPCR. Ethidium bromide stained gel after amplification of the CVS11 strain using dilutions from $10^{-1}$ to $10^{-8}(\mathrm{~A}-\mathrm{H})$. The amplification product of $582 \mathrm{bp}$ is clearly visible up to a dilution of $10^{-5}$ (lane E). Ladder $=$ standard for determination of amplification product size.

3.2. Sensitivity of PCR Methods. For the comparison with infectious titres, RNA of the classical rabies virus strain CVS11 was extracted from cell culture supernatants (BHK-21 cells or neuroblastoma cells MNA 42/13) and serially diluted from $10^{-1}$ to $10^{-8}$. The infectious titre was determined according to the Spearman-Karber formula $[49,50]$ in fluorescent focus forming doses $50 \%\left(\mathrm{FFD}_{50}\right)$. In the heminested RT-PCR (hnRT-PCR), the amplification product of $582 \mathrm{bp}$ was visible up to a dilution of $10^{-5}$ corresponding to a detection limit of $0.4 \mathrm{FFD}_{50}\left(10^{-0.4} \mathrm{FFD}_{50}\right.$; Figure 1). In the real-time RT-PCR (NDWD) the serial dilution was performed in triplicate. The limit of detection was defined as the last dilution at which at least 2 out of 3 replicates were positive. Using this definition, CVS-11 was detectable up to a dilution of $10^{-7}$ (once at a dilution of $10^{-8}$ ). The amplification plot showed regular intervals of the curves with CT-values from 18.1 (dilution $10^{-1}$ ) to 40.0 (dilution $10^{-7}$ ). Relating to infectious titre realtime RT-PCR reached a detection limit of $0.003 \mathrm{FFD}_{50}\left(10^{-2.6}\right)$ for CVS-11.

The efficiency of the real-time RT-PCR (NDWD) was determined using linear regression of the CT-values and the titres of the samples (Figure 2). On the basis of the slope coefficient, the efficiency can be inversely derived as $E=$ $10^{(-1 / \text { slope coefficient })}-1$. The efficiency for CVS-11 was $94.5 \%$ with a slope coefficient of -3.46 .

3.3. Analytical Sensitivity. The limit of detection in terms of DNA copy numbers was determined using 10-fold serial dilutions of a cloned 933 bp segment of $\mathrm{N}$ of CVS-11 containing $10^{6}$ to $10^{0} \mathrm{DNA}$ copies/PCR reaction. In the hnPCR, the limit of detection was 100 DNA copies/ $3 \mu \mathrm{L}$ of starting material of the CVS-11 plasmid. In the real-time PCR (NDWD), the 10fold serial dilution of the CVS-plasmid was tested in triplicate. The limit of detection was 10 DNA copies $/ 2 \mu \mathrm{L}$ of the CVS-11 plasmid with CT-values between 38.6 and 47.5 . The efficiency was at $82.8 \%$ with a slope coefficient of -3.82 (not shown). The intra-assay repeatability of the real-time PCR (NDWD)

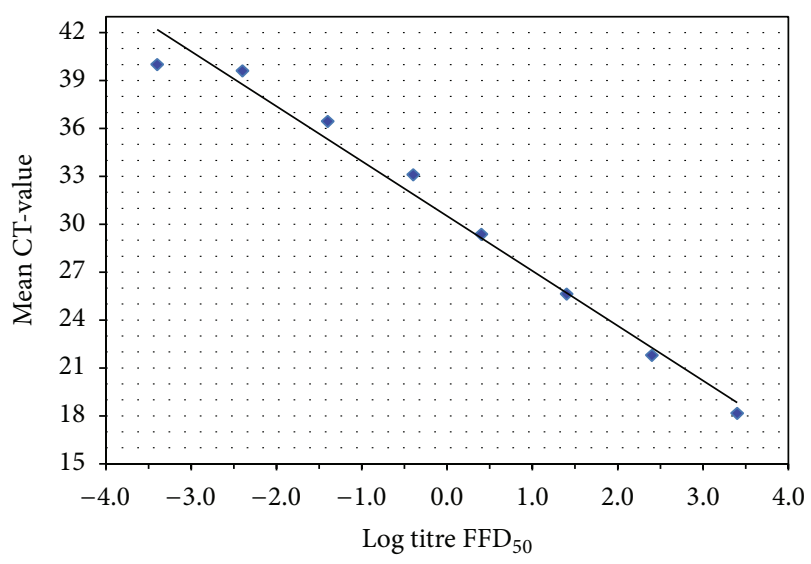

FIgURE 2: Efficiency of the real-time RT-PCR (NDWD). The linear regression of the CT-values (ordinate) and the titres of serially diluted CVS-11 (abscissa) exhibited a slope coefficient of -3.46 corresponding to an efficiency of $94.5 \%\left(E=10^{(-1 / \text { slope coefficient })}-1\right)$.

proved to be excellent with very low coefficients of variation up to $10^{2}$ DNA copies $(0.18-1.37 \%)$ with and outlier at $10^{1}$ copies (12.05\%; Table 3).

3.4. Diagnostic Broadness of PCR Methods. A total of 31 lyssaviruses (19 identified as classical RABV, 7 as EBLV1,4 as EBLV-2, and 1 as DUVV) were used to test the diagnostic performance of the PCR (Table 1). All viruses used were detected with the expected band size in the hnRTPCR technique (Table 4). The specificity of the amplified product was confirmed as RABV, EBLV-1, EBLV-2, or DUVV by sequencing in each case. Host GAPDH (glyceraldehyde 3-phosphate dehydrogenase) was detected in all of the cell culture supernatants and mouse brain suspensions as internal control, indicating that sample material was present and RNA isolation, reverse transcription, and amplification were not inhibited. All samples were also tested positive by the real-time RT-PCR assay (Table 4). Sendai virus as internal control for inhibition was run in parallel using the same cycling conditions. In the real-time RT-PCR (NDWD) with an annealing temperature of $50^{\circ} \mathrm{C}$, some samples were detected with more than one probe. With the exception of the sample derived from a raccoon dog from Poland, the probe for the homologous genotype always yielded the lowest CT-value. In this exceptional case, the probe for the detection of genotype 6 was slightly lower than the one for genotype 1 (15.0 versus 16.1; Table 4). Comparison of these two probes with the sequence of the rabies variant in question showed 1 and 2 mismatches with the RABVD1$\mathrm{P}$ and LysGT6-P probe, respectively (Figure 3). Duvenhage virus, also designated as genotype 4 , was detectable with the probe adapted for genotype 6 in spite of 3 mismatches (LysGT6-P). The probe LysGT6-P was able to detect synthetic DNA fragments of BBLV, ARAV, and IRKV, although containing one or two mismatches, respectively. The synthetic DNA fragments of KHUV, ABLV, LBV, MOKV, SHIBV, and WCBV, which exhibited from four (ABLV/KHUV) up to ten (WCBV) mismatches to the best fitting probe for GT1, 
TABLE 3: Serial dilutions of the CVS-11 plasmid in real-time PCR (NDWD).

\begin{tabular}{|c|c|c|c|c|c|c|}
\hline Dilution & Copy numbers $/ 2 \mu \mathrm{L}$ & CT-value 1 & CT-value 2 & CT-value 3 & Mean \pm SD & $\mathrm{CV}(\%)$ \\
\hline $10^{-1}$ & $10^{6}$ & 22.6 & 22.0 & 22.4 & $22.3 \pm 0.31$ & 1.37 \\
\hline $10^{-2}$ & $10^{5}$ & 25.1 & 25.2 & 25.3 & $25.2 \pm 0.10$ & 0.40 \\
\hline $10^{-3}$ & $10^{4}$ & 29.1 & 28.8 & 28.7 & $28.9 \pm 0.21$ & 0.72 \\
\hline $10^{-4}$ & $10^{3}$ & 32.9 & 32.9 & 32.8 & $32.9 \pm 0.06$ & 0.18 \\
\hline $10^{-5}$ & $10^{2}$ & 36.4 & 36.2 & 35.9 & $36.2 \pm 0.25$ & 0.70 \\
\hline $10^{-6}$ & $10^{1}$ & 47.5 & 38.6 & 39.0 & $41.7 \pm 5.03$ & 12.05 \\
\hline $10^{-7}$ & $10^{0}$ & - & - & - & - & - \\
\hline
\end{tabular}

SD: standard deviation; $\mathrm{CV}$ : coefficient of variation.

TABLE 4: PCR-results of rabies viruses tested.

\begin{tabular}{|c|c|c|c|c|c|c|}
\hline & & & & & e RT-PCR ( & \\
\hline & Sample & Species & hnRT-PCR & & CT-value ${ }^{a}$ & \\
\hline & & & & FAM (GT1) & YY (GT5) & Cy5 (GT6) \\
\hline 1 & Bat Stade (1970) & EBLV-1 & + & & 14.7 & 17.3 \\
\hline 2 & Bat Hamburg RV 9 (1968) & EBLV-1 & + & 20.2 & 14.8 & 16.4 \\
\hline 3 & Bat Finland RV 8 (1986) & EBLV-2 & + & & & 15.1 \\
\hline 4 & Bat Holland RV 31 & EBLV-1 & + & 20.2 & 14.7 & 16.8 \\
\hline 5 & Dog Tunisia (1986) & RABV & + & 24.6 & 27.2 & 33.2 \\
\hline 6 & Bat Spain RV 119 & EBLV-1 & + & 33.4 & 27.6 & 31.6 \\
\hline 7 & Bat Florida & RABV & + & 24.2 & 41.6 & \\
\hline 8 & Bat Denmark & EBLV-1 & + & 26.5 & 21.1 & 22.4 \\
\hline 9 & Raccoon dog Poland (1985) & RABV & + & 16.1 & & 15.0 \\
\hline 10 & Raccoon Florida (1986) & RABV & + & 25.8 & & \\
\hline 11 & Bat Chile RV 108 & RABV & + & 14.4 & & \\
\hline 12 & Jackal Zimbabwe & RABV & + & 14.6 & & \\
\hline 13 & Cattle Zimbabwe & RABV & + & 18.0 & & \\
\hline 14 & Canada Red Fox & RABV & + & 17.9 & & \\
\hline 15 & LEP (Flury, 1939) & RABV & + & 17.7 & & \\
\hline 16 & HEP (Flury) & RABV & + & 28.5 & & \\
\hline 17 & Dog Nepal Nr. 96 & RABV & + & 21.7 & & \\
\hline 18 & Sri Lanka dog 121 (1986) & RABV & + & 23.2 & & \\
\hline 19 & Eurofox $912 / 87$ & RABV & + & 28.3 & & \\
\hline 20 & Duvenhage RV6 & DUVV & + & & & 27.0 \\
\hline 21 & NYC 58 & RABV & + & 16.5 & & \\
\hline 22 & Dog Lima & RABV & + & 20.0 & & \\
\hline 23 & Bat Bremerhaven RV11 & EBLV-1 & + & & 27.0 & \\
\hline 24 & Bat B24 & EBLV-1 & + & & 16.9 & 20.6 \\
\hline 25 & Skunk Canada & RABV & + & 23.2 & & \\
\hline 26 & SAD Bern, 1935 & RABV & + & 18.8 & & \\
\hline 27 & CSSR/A-virus & RABV & + & 18.5 & & \\
\hline 28 & Challenge virus standard (CVS-11) ATCC VR 959 & RABV & + & 19.8 & & \\
\hline 29 & TW 1814/92 & EBLV-2 & + & & & \\
\hline 30 & TW 1392/93 & EBLV-2 & + & & & \\
\hline 31 & TW $118 / 02$ & EBLV-2 & + & & & \\
\hline
\end{tabular}

${ }^{\mathrm{a}}$ Only for samples with positive reaction.

FAM: 6-carboxyfluorescein reporter dye, to detect RABV (GT1); YY: Yakima Yellow, to detect EBLV-1 (GT5); Cy5: Cyanine 5, to detect EBLV-2 (GT6); ND: not done. 

RABVD1-P $\quad 5^{\prime}-78$ ccgayaagattgtattyaargtcaakaatcaggt $111-3^{\prime}$
RABV raccoon $\operatorname{dog} \quad \ldots$.................c.t.......

(a)
LysGT6-P $\quad 5^{\prime}-81$ acaraattgtcttcaargtccataatcag 109-3' RABV raccoon dog ...ag....................

(b)

Figure 3: Alignment of probes RABVD1-P (a) and LysGT6-P (b) with a RABV strain isolated from a raccoon dog from Poland. Matches in nondegenerated positions are displayed as dots. Matches in wobbled positions are highlighted in yellow. Mismatches are highlighted in turquoise.

GT6, or GT5, respectively, were all detected with the corresponding species-specific new probe (Table 2). $10^{5.2}$ copies of the synthetic DNA were detected at CT-values of 27.933.5 for ABLV, MOKV, SHIBV, and WCBV (not shown). The efficiency of detection of KHUV, which showed an atypical amplification plot at the highest number of $10^{10.2}$ copies of the synthetic DNA, and that of LBV was lower with CTvalues of 40.0 and 40.5 at a copy number of $10^{5.2}$, respectively (not shown). Multiplexing of combinations of these probes (ivvLBV-P (FAM: 6-carboxyfluorescein), ivvKhujand-P (YY: Yakima Yellow), and ivvSBLV2-P (Cy5: Cyanine 5), as well as ivvWCBV-P (FAM), ivvMok-P (YY), and ivvABLV2n-P (Cy5)) as for the species 1,5, and 6 was not successful.

\subsection{Sequencing of Amplification Products and Phylogenetic} Analysis. The $543 \mathrm{bp}$ amplification products of the nucleoprotein gene obtained in the heminested PCR reactions were sequenced (Table 1) and analysed phylogenetically with additional sequences retrieved from GenBank representing all known lyssaviruses. The similarity of the $543 \mathrm{bp}$ nucleotide sequences of the nucleoprotein gene (positions 74-616 according to the Pasteur virus genome) among the lyssaviruses included ranged from $64.6 \%$ to $90.1 \%$ (Hamming distance). Similarity at the amino acid level was $68.0-95 \%$. The resulting phylogenetic tree obtained by the neighborjoining method implemented in the MEGA5 software is presented in Figure 4. All known genotypes were resolved with high bootstrap confidence with our samples grouping expectedly.

3.6. Clinical Specimens. Clinical specimens like brain suspensions (1x human, 12x mouse), skin biopsies from the nape of the neck ( $3 x$ human, $1 x$ mouse), saliva ( $4 x$ human), and cerebrospinal fluids (6x human) were used for the establishment of the assays. Both hnRT-PCR and real-time RT-PCR (NDWD) were shown to work properly on all these samples without significant inhibition using GAPDH as internal control for conventional RT-PCR and Sendai virus for real-time RT-PCR (NDWD), which was mixed to the samples before RNA isolation. Skin biopsies taken from the neck and lip of a mouse (white Swiss mouse at an age of 3 weeks) euthanized 2 weeks after intracerebral infection with SAD Bern virus 20 years ago were positive with the hnRTPCR and real-time PCR (NDWD). Skin biopsies taken from other locations on the head were positive with the real-time PCR (NDWD) and weakly positive in hnRT-PCR whereas samples taken around the vibrissae were weakly positive in real-time PCR (NDWD) only. Sequencing of amplification products excluded a contamination with the positive CVS-11 control (not shown).

Brain suspension from an imported human rabies case in 2012 was already strongly positive after one round of the hnRT-PCR. Phylogenetic analysis of the $543 \mathrm{bp}$ nucleotide fragment revealed its close relationship to the classical rabies virus strains circulating in the insectivorous Mexican freetailed bat, Tadarida brasiliensis, a species common in the southern United States and Mexico. This allowed identification of the origin of the patient's infection, who had travelled extensively and did not report any previous biting incident $[51,52]$.

\section{Discussion}

Based on a large amount of published work, we were able to establish and quantitatively characterise RT-PCR protocols for the detection of lyssaviruses in clinical samples. Well suited real-time protocols $[8,25]$ for the detection of classical rabies virus and the genotypes 5 and 6 (EBLV-1 and EBLV-2) were adapted and extended for the detection of at least 13 lyssavirus species. To this goal, 6 speciesspecific probes (KHUV, ABLV, LBV, MOKV, SHIBV, and WCBV) were designed and verified on synthetic DNA fragments encompassing the targeted sequence of the lyssaviral nucleoprotein gene. Multiplexing the probes in a single-tube reaction as described for the genotypes 1, 5, and 6 was not possible with the newly designed probes, probably due to false priming and/or interference within the mix of reagents and synthetic target sequence [53]. As far as evaluated with the multiplexed probes for the genotypes 1, 5, and 6 using 31 viral isolates, which were all detectable with high sensitivity, direct differentiation of targeted genotypes on the base of the quantitative reaction (CT-values) was mostly possible. Furthermore, a single probe for genotype 6 (species EBLV-2) was able to detect up to 7 genotypes/species. We consider this type of cross hybridization as an advantage of the technique using degenerate primers and probes in terms of a broad detectability of lyssavirus rather than a lack of accuracy of discrimination. In an approach using SYBR Green qPCR with similarly degenerate primers spanning the same part of $\mathrm{N}$ as in this work [28], the detection of all lyssavirus species known at that time was achieved. Using several genotypespecific probes in a TaqMan real-time protocol, we were able to add more intrinsic confidence to the specificity of the analysis [54]. Quantitative characterisation of the assay using the probe for genotypes 1, 5, and 6 on CVS-11 showed excellent sensitivity and repeatability with a detection limit of as low as an infectious dose of $0.003 \mathrm{FFD}_{50}$ combined 


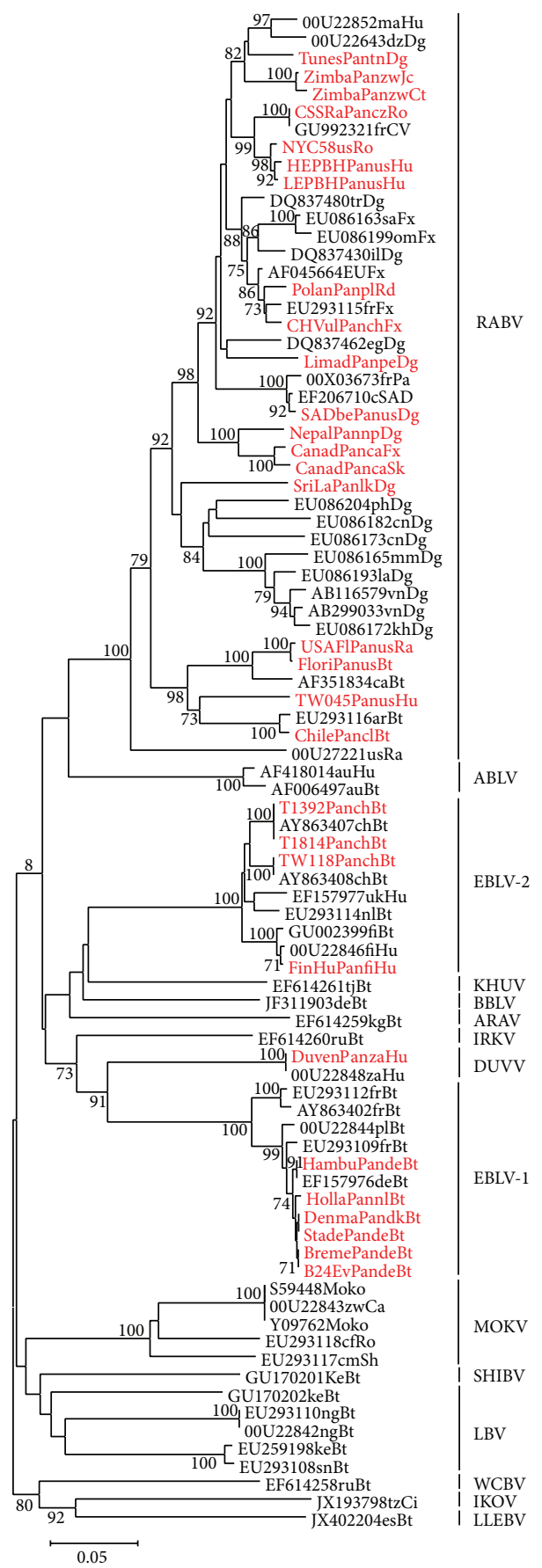

FIGURE 4: Phylogenetic tree with 543 bp fragments of N. Phylogenetic tree obtained with MEGA5 software [35]. The length of branches (horizontal lines) corresponds to phylogenetic distance between different sequences (scale bar in substitutions per site). Numbers proximal to nodes indicate bootstrap confidence of subjacent groups. Lyssavirus strains used in this study are depicted in red. Sequences are given with GenBank or own designation followed by the two-letter country code and a two-letter code for the source species as follows: Bt, bat; Ca, cat; Ci, African civet; Dg, dog; Fx, fox; Hu, human; Jc, jackal; Ra, raccoon; Rd, raccoon dog; Ro, rodent; Sh, shrew; Sk, skunk, except for Pa, cSAD, and CV, which are standing for Pasteur strain, Street Alabama Dufferin strain, and challenge virus standard, respectively. The currently used abbreviations for species are shown next to the tree. RABV, rabies virus; ABLV, Australian bat lyssavirus; WCBV, West Caucasian bat virus; IKOV, Ikoma virus; LLEBV, Lleida bat lyssavirus; MOKV, Mokola virus; SHIBV, Shimoni bat virus; LBV, Lagos bat virus; KHUV, Khujand virus; BBLV, Bokeloh bat lyssavirus; ARAV, Aravan virus; DUVV, Duvenhage virus; EBLV, European bat lyssavirus; IRKV, Irkut virus. 
with an analytical sensitivity of 10 DNA copies at efficiencies of $94.5 \%$ and $82.8 \%$, respectively. Considering the amount of degeneration in both primers and probes, the efficiencies determined must be considered satisfactory. As far as the absolute detection limit in terms of target copies is concerned, the usage of plasmid DNA rather than RNA must be kept in mind. Since reverse transcription cannot be assumed as $100 \%$ efficient and reproducible [55], the limit for RNA might be somewhat lower.

For further characterisation of samples with a positive reaction in real-time RT-PCR, excellent simple and (hemi)nested RT-PCR protocols are available. With the protocol used for this work [22], all 31 viral strains used could be amplified and sequenced, confirming the diagnostic broadness of the assay. Phylogenetic analysis of these partial nucleotide sequences belonging to genotypes $1,4,5$, and 6 along with known sequences confirmed the suitability of this relatively conserved genomic region for molecularepidemiological characterisation of lyssaviruses circulating worldwide [56-58]. This was also confirmed in the context of a human rabies case imported to Switzerland in 2012, which could be attributed unequivocally to an exposure to a bat of the species Tadarida brasiliensis in California, USA.

\section{Conclusion}

In this work we could show and validate the suitability of an adapted and further developed real-time RT-PCR protocol for the rapid, efficient, and highly sensitive intravitam diagnosis of a wide spectrum of lyssavirus species followed by rapid molecular-epidemiological characterisation of viral isolates respective to origin of the virus and source of exposure, using heminested RT-PCR. This new tool is also particularly promising for active surveillance of European bat lyssaviruses using oral swabs in live-captured bats.

\section{Conflict of Interests}

The authors declare that there is no conflict of interests regarding the publication of this paper.

\section{Acknowledgments}

The authors wish to acknowledge the critical review of the paper of PD Dr. Philippe Plattet. The help of Audrey Megali for her advice in the molecular techniques is greatly acknowledged.

\section{References}

[1] Laboratory Techniques in Rabies, World Health Organization, Geneva, Switzerland, 4th edition, 1996.

[2] WHO, "WHO expert consultation on rabies," Second Report, WHO, Geneva, Switzerland, 2013.

[3] O. I. E. Rabies, Manual of Standards for Diagnostic Tests and Vaccines for Terrestrial Animals, World Health Organization, Paris, France, 2013.

[4] D. Sacramento, H. Bourhy, and N. Tordo, "PCR technique as an alternative method for diagnosis and molecular epidemiology of rabies virus," Molecular and Cellular Probes, vol. 5, no. 3, pp. 229-240, 1991.

[5] H. Bourhy, P. Sureau, and J. A. Montano Hirose, Méthodes de Laboratoire pour le Diagnostic de la Rage, Institut Pasteur, Paris, France, 1990.

[6] E. Picard-Meyer, V. Bruyère, J. Barrat, E. Tissot, M. J. Barrat, and F. Cliquet, "Development of a hemi-nested RT-PCR method for the specific determination of European Bat Lyssavirus 1: comparison with other rabies diagnostic methods," Vaccine, vol. 22, no. 15-16, pp. 1921-1929, 2004.

[7] T. Nagaraj, J. P. Vasanth, A. Desai, A. Kamat, S. N. Madhusudana, and V. Ravi, "Ante mortem diagnosis of human rabies using saliva samples: comparison of real time and conventional RT-PCR techniques," Journal of Clinical Virology, vol. 36, no. 1, pp. 17-23, 2006.

[8] S. A. Nadin-Davis, M. Sheen, and A. I. Wandeler, "Development of real-time reverse transcriptase polymerase chain reaction methods for human rabies diagnosis," Journal of Medical Virology, vol. 81, no. 8, pp. 1484-1497, 2009.

[9] S. Wacharapluesadee and T. Hemachudha, "Ante- and postmortem diagnosis of rabies using nucleic acid-amplification tests," Expert Review of Molecular Diagnostics, vol. 10, no. 2, pp. 207-218, 2010.

[10] M. Fischer, K. Wernike, C. M. Freuling et al., "A step forward in molecular diagnostics of lyssaviruses-results of a ring trial among European laboratories," PLoS ONE, vol. 8, no. 3, Article ID e58372, 2013.

[11] C. Freuling, A. Vos, N. Johnson et al., "Experimental infection of serotine bats (Eptesicus serotinus) with European bat lyssavirus type 1a," Journal of General Virology, vol. 90, no. 10, pp. 24932502, 2009.

[12] K. Schutsky, D. Curtis, E. K. Bongiorno et al., "Intramuscular inoculation of mice with the live-attenuated recombinant rabies virus TriGAS results in a transient infection of the draining lymph nodes and a robust, long-lasting protective immune response against rabies," Journal of Virology, vol. 87, no. 3, pp. 1834-1841, 2013.

[13] H. Bourhy, J.-M. Reynes, E. J. Dunham et al., "The origin and phylogeography of dog rabies virus," Journal of General Virology, vol. 89, no. 11, pp. 2673-2681, 2008.

[14] D. T. S. Hayman, N. Johnson, D. L. Horton et al., "Evolutionary history of rabies in Ghana," PLoS Neglected Tropical Diseases, vol. 5, no. 4, Article ID e1001, 2011.

[15] S. A. Nadin-Davis and L. A. Real, "Molecular phylogenetics of the lyssaviruses-insights from a coalescent approach," Advances in Virus Research, vol. 79, pp. 203-238, 2011.

[16] C. A. Hanlon and J. E. Childs, "Epidemiology," in Rabies: Scientific Basis of Disease and its Management, A. C. Jackson, Ed., pp. 61-121, Academic Press, New York, NY, USA, 2013.

[17] S. A. Nadin-Davis, "Molecular epidemiology," in Rabies: Scientific Basis of Disease and Its Management, A. C. Jackson, Ed., pp. 123-177, Academic press, Amsterdam, The Netherlands, 2013.

[18] P. R. Heaton, P. Johnstone, L. M. McElhinney, R. Cowley, E. O'Sullivan, and J. E. Whitby, "Heminested PCR assay for detection of six genotypes of rabies and rabies-related viruses," Journal of Clinical Microbiology, vol. 35, no. 11, pp. 2762-2766, 1997.

[19] H. Bourhy, "Lyssaviruses—special emphasis on rabies virus," in Diagnostic Virology Protocols, J. R. Stephenson and A. Warnes, Eds., vol. 12 of Methods in Molecular Medicine, pp. 129-142, Humana Press, Totowa, NJ, USA, 1998. 
[20] F. Cliquet and E. Picard-Meyer, "Rabies and rabies-related viruses: a modern perspective on an ancient disease," Revue Scientifique et Technique de L'Office International des Epizooties, vol. 23, no. 2, pp. 625-642, 2004.

[21] S. Vázquez-Morón, A. Avellón, and J. E. Echevarría, "RT-PCR for detection of all seven genotypes of Lyssavirus genus," Journal of Virological Methods, vol. 135, no. 2, pp. 281-287, 2006.

[22] M. Panning, S. Baumgarte, S. Pfefferle, T. Maier, A. Martens, and C. Drosten, "Comparative analysis of rabies virus reverse transcription-PCR and virus isolation using samples from a patient infected with rabies virus," Journal of Clinical Microbiology, vol. 48, no. 8, pp. 2960-2962, 2010.

[23] P. de Benedictis, C. de Battisti, L. Dacheux et al., "Lyssavirus detection and typing using pyrosequencing," Journal of Clinical Microbiology, vol. 49, no. 5, pp. 1932-1938, 2011.

[24] E. M. Black, J. P. Lowings, J. Smith, P. R. Heaton, and L. M. McElhinney, "A rapid RT-PCR method to differentiate six established genotypes of rabies and rabies-related viruses using TaqMan (TM) technology," Journal of Virological Methods, vol. 105, pp. 25-35, 2002.

[25] P. R. Wakeley, N. Johnson, L. M. McElhinney, D. Marston, J. Sawyer, and A. R. Fooks, "Development of a real-time, TaqMan reverse transcription-PCR assay for detection and differentiation of lyssavirus genotypes 1,5, and 6," Journal of Clinical Microbiology, vol. 43, no. 6, pp. 2786-2792, 2005.

[26] J. Coertse, J. Weyer, L. H. Nel, and W. Markotter, "Improved PCR methods for detection of african rabies and rabies-related lyssaviruses," Journal of Clinical Microbiology, vol. 48, no. 11, pp. 3949-3955, 2010.

[27] B. Hoffmann, C. M. Freuling, P. R. Wakeley et al., "Improved safety for molecular diagnosis of classical rabies viruses by use of a TaqMan real-time reverse transcription-PCR "double check" strategy," Journal of Clinical Microbiology, vol. 48, no. 11, pp. 3970-3978, 2010.

[28] D. T. S. Hayman, A. C. Banyard, P. R. Wakeley et al., "A universal real-time assay for the detection of Lyssaviruses," Journal of Virological Methods, vol. 177, no. 1, pp. 87-93, 2011.

[29] T. Müller, N. Johnson, C. M. Freuling, A. R. Fooks, T. Selhorst, and A. Vos, "Epidemiology of bat rabies in Germany," Archives of Virology, vol. 152, no. 2, pp. 273-288, 2007.

[30] A. C. Banyard, N. Johnson, K. Voller et al., "Repeated detection of European bat lyssavirus type 2 in dead bats found at a single roost site in the UK," Archives of Virology, vol. 154, no. 11, pp. 1847-1850, 2009.

[31] A. Megali, G. Yannic, M.-L. Zahno et al., "Surveillance for European bat lyssavirus in Swiss bats," Archives of Virology, vol. 155, no. 10, pp. 1655-1662, 2010.

[32] W. W. Müller, "Review of reported rabies case data in Europe to the WHO Collaborating Centre, Tübingen, from 1977 to 1988," Rabies Bulletin Europe, vol. 4, pp. 16-19, 1977.

[33] C. Freuling, T. Selhorst, A. Kliemt, and T. Müller, "Rabies surveillance in Europe, 2004-2007," Rabies Bulletin Europe, vol. 31, no. 4, pp. 7-8, 2004.

[34] P. Demetriou and J. Moynagh, "The European Union strategy for external cooperation with neighbouring countries on rabies control," Rabies Bulletin Europe, vol. 35, no. 1, pp. 5-7, 2011.

[35] K. Tamura, D. Peterson, N. Peterson, G. Stecher, M. Nei, and S. Kumar, "MEGA5: molecular evolutionary genetics analysis using maximum likelihood, evolutionary distance, and maximum parsimony methods," Molecular Biology and Evolution, vol. 28, no. 10, pp. 2731-2739, 2011.
[36] M. K. Abelseth, "An attenuated rabies vaccine for domestic animals produced in tissue culture," Canadian Veterinary Journal, vol. 5, no. 11, pp. 279-286, 1964.

[37] A. P. Ravazzolo, C. Nenci, H.-R. Vogt et al., "Viral load, organ distribution, histopathological lesions, and cytokine mRNA expression in goats infected with a molecular clone of the caprine arthritis encephalitis virus," Virology, vol. 350, no. 1, pp. 116-127, 2006.

[38] D. Kaiser, Entwicklung und Evaluation eines RT-TaqMan-PCRTestverfahrens zum Nachweis des BVD-Virus [Ph.D. dissertation], Universität Bern, 2001.

[39] H. Koprowski, "The mouse inoculation test," in Laboratory Techniques in Rabies, M. M. Kaplan and H. Koprowski, Eds., pp. 85-93, World Health Organization, Geneva, Switzerland, 1973.

[40] D. J. Dean and M. K. Abelseth, "The fluorescent antibody test," in Laboratory Techniques in Rabies, M. M. Kaplan and H. Koprowski, Eds., pp. 73-83, World Health Organization, Geneva, Switzerland, 1973.

[41] A. Gerhardt, Teilvalidierung einer Zellkulturmethode als Alternative zum Tierversuch für den Nachweis von Tollwutvirus aus Hirnmaterial [dissertation thesis], Veterinärmedizinische Universität Wien, Universität Bern, 1995.

[42] R. J. Rudd and C. V. Trimarchi, "Development and evaluation of an in vitro virus isolation procedure as a replacement for the mouse inoculation test in rabies diagnosis," Journal of Clinical Microbiology, vol. 27, no. 11, pp. 2522-2528, 1989.

[43] P. Stöhr, K. Stöhr, H. Kiupel, and E. Karge, "Immunofluorescence investigations of rabies field viruses in comparison of several fluorescein-labelled antisera," Tierärztliche Umschau, vol. 47, pp. 813-818, 1992.

[44] J. D. Thompson, T. J. Gibson, F. Plewniak, F. Jeanmougin, and D. G. Higgins, “The CLUSTAL X windows interface: flexible strategies for multiple sequence alignment aided by quality analysis tools," Nucleic Acids Research, vol. 25, no. 24, pp. 48764882, 1997.

[45] D. G. Higgins and P. M. Sharp, "CLUSTAL: a package for performing multiple sequence alignment on a microcomputer," Gene, vol. 73, no. 1, pp. 237-244, 1988.

[46] M. A. Larkin, G. Blackshields, N. P. Brown et al., "Clustal W and clustal X version 2.0," Bioinformatics, vol. 23, no. 21, pp. 29472948, 2007

[47] T. Maniatis, E. F. Fritsch, and J. Sambrook, Molecular Cloning: A Laboratory Manual, Cold Spring Harbor Laboratory, Cold Spring Harbor, NY, USA, 1982.

[48] K. B. Nicholas, H. B. J. Nicholas, and D. W. Deerfield II, "GeneDoc: analysis and visualization of genetic variation," Embnew News, vol. 4, article 14, 1997.

[49] G. Kaerber, "Beitrag zur kollektiven Behandlung pharmakologischer Reihenversuche," Archiv for Experimentelle Pathologie und Pharmakologie, vol. 162, pp. 480-487, 1931.

[50] C. Spearman, "The method of "right or wrong cases" (constant stimuli) without Gauss's formulae," British Journal of Psychology, vol. 2, pp. 227-242, 1908.

[51] A. N. Deubelbeiss, C. Trachsel, E. B. Bachli et al., "Imported human rabies in Switzerland, 2012: a diagnostic conundrum," Journal of Clinical Virology, vol. 57, no. 2, pp. 178-181, 2013.

[52] S. Farley, S. Zarate, E. Jenssen et al., "U.S-acquired human rabies with symptom onset and diagnosis abroad, 2012," Morbidity and Mortality Weekly Report, vol. 61, no. 39, pp. 777-781, 2012.

[53] A. Apte and S. Daniel, PCR Primer Design, Cold Spring Harbor Protocols, 2009. 
[54] C. J. Smith and A. M. Osborn, "Advantages and limitations of quantitative PCR (Q-PCR)-based approaches in microbial ecology," FEMS Microbiology Ecology, vol. 67, no. 1, pp. 6-20, 2009.

[55] R. Sanders, D. J. Mason, C. A. Foy, and J. F. Huggett, "Evaluation of digital PCR for absolute RNA quantification," PLOS ONE, vol. 8, no. 9, Article ID e75296, 2013.

[56] E. C. Holmes, C. H. Woelk, R. Kassis, and H. Bourhy, "Genetic constraints and the adaptive evolution of rabies virus in nature," Virology, vol. 292, no. 2, pp. 247-257, 2002.

[57] A. J. Foord, H. G. Heine, L. I. Pritchard et al., "Molecular diagnosis of lyssaviruses and sequence comparison of Australian bat lyssavirus samples," Australian Veterinary Journal, vol. 84, no. 7, pp. 225-230, 2006.

[58] T. Scott, R. Hassel, and L. Nel, "Rabies in kudu (Tragelaphus strepsiceros)," Berliner und Munchener Tierarztliche Wochenschrift, vol. 125, no. 5-6, pp. 236-241, 2012. 

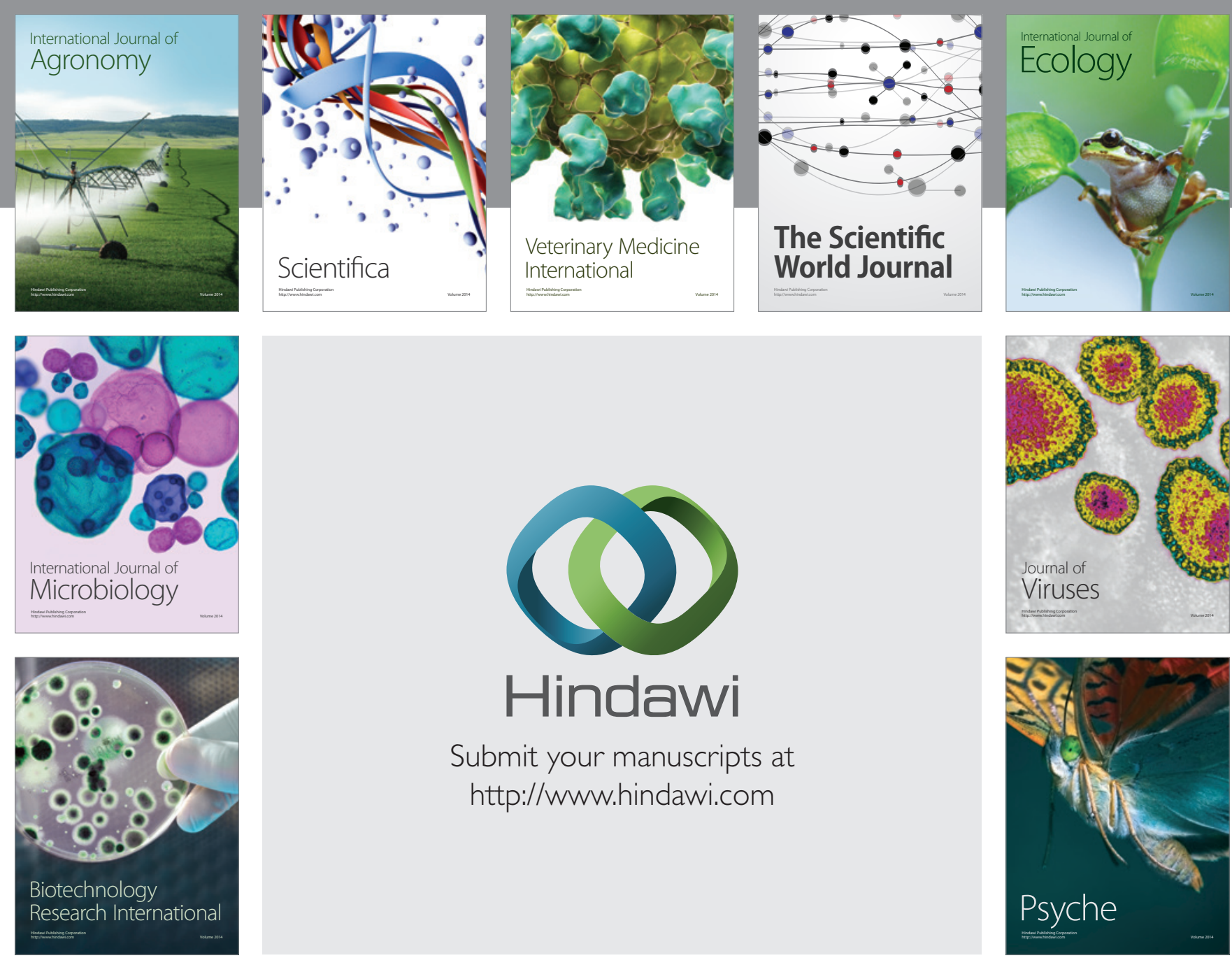

Submit your manuscripts at http://www.hindawi.com
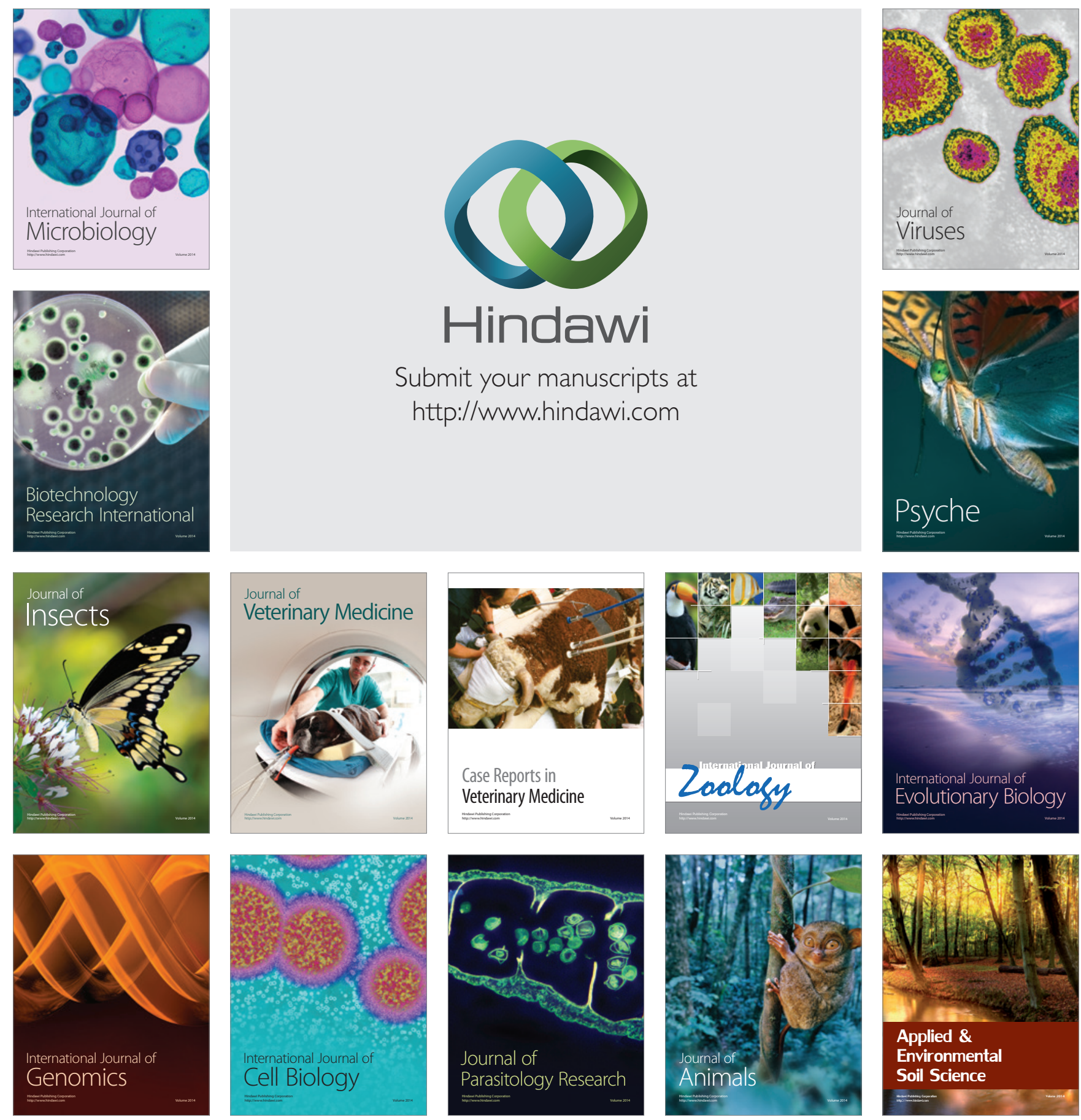